\title{
Combined Subbrow Excision with Upper Blepharoplasty Using a Tadpole Shaped Excision for Blepharochalasis
}

\author{
Hwa Young Oh, In Ho Lee, \\ Soo Chung Hong, Syeo Young Wee, \\ Chang Yong Choi \\ Department of Plastic and Reconstructive \\ Surgery, College of Medicine, \\ Soonchunhyang University, Gumi \\ Hospital, Gumi, Korea
}

This work was supported by the Soonchunhyang University Research Fund.

No potential conflict of interest relevant to this article was reported.
Background In efforts to solve blepharochalasis, induced by aging process, various surgical methods have been developed over the years. Among them, classical upper blepharoplasty has a problem that severe lateral hooding may be restricted in making correction, cause of difference in thickness between the pretarsal skin and subbrow skin. And also subbrow excision (SE) blepharoplasty cannot be easily corrected in severe drooping of the medial skin. In an effort to make up for the disadvantages of these methods, we attempted SE-tadpole shaped excision blepharoplasty (TSEB).

Methods Fifteen patients underwent SE-TSEB from January 2013 to November 2014. SE-TSEB was performed the following cases: 1 ) patients who showed moderate to severe blepharochasis with severe lateral wrinkles; 2) patients with a history of pre-existent SE blepharoplasty; 3) patients with a tattoo on the eyebrows, who want to make revision; and 4) patients with eyelid skins that are thick or have overly protuberant fat pads of the retro-orbicularis oculi fat (ROOF), and have a plan to extirpate them.

Results During the follow-up observation period, most patients showed amicable correction of visibility difficulty and were aesthetically satisfied with the postoperative results with respect to correction of the lateral wrinkles. Scars formed in the subbrow incision line were within the scope of management.

Conclusions SE-TSEB is a treatment modality that overcomes the disadvantages and limitations of classical upper blepharoplasty and SE blepharoplasty. Furthermore, maximizing the advantage of each surgical approach is another innovative approach for correction of changes in the upper eyelid, induced by aging.

Keywords : Blepharochalasis, Blepharoplasty, Eyelids

\section{INTRODUCTION}

In efforts to solve blepharochalasis, the problem of droopy eyelids that happens as people age, various surgical methods have been developed and disclosed over the years. Among them, classical upper blepharoplasty, the most fundamental procedure, involves exci-

Received: Jan 14, 2015 Revised: Feb 2, 2015 Accepted: Feb 3, 2015 Correspondence: Chang Yong Choi Department of Plastic and Reconstructive Surgery, Soonchunhyang University Gumi Hospital, 179 1gongdan-ro, Gumi 730-706, Korea. E-mail: 73120@schmc.ac.kr

Copyright @ 2015 The Korean Society for Aesthetic Plastic Surgery.

This is an Open Access article distributed under the terms of the Creative Commons Attribution Non-Commercial License (http://creativecommons.org/licenses/by-nc/3.0/) which permits unrestricted non-commercial use, distribution, and reproduction in any medium, provided the original work is properly cited. www.e-aaps.org sion of redundant eyelid skin in the upper palpebrae [1-4]. This operation utilizes an approach through the lid crease line of the upper eyelids, and hence excess skin can be excised relatively evenly throughout the entire eyelid. Also aponeurotic ptosis can be corrected by aponeurotic surgery through this incision opening $[5,6]$. Furthermore, new double eyelids may be created, or the width of a preexisting double eyelid may be adjusted, by either the surgeon in charge or the demand of a patient. Nevertheless, postoperative appearance may look unnatural and severe lateral hooding may be restricted in making correction. Moreover, owing to differences in thickness between the pretarsal skin and subbrow skin, it may lead to an appearance of the eyelids being seen as overhanging and protuberant $[7,8]$. Subbrow excision (SE) blepharoplasty has been performed to improve on the disadvantage of classical upper blepharoplasty. Parkes et al.[9] first introduced this procedure in the mid 
1970s. This surgical approach can resolve the disadvantage of classical upper blepharoplasty by extirpating excessive skin, subcutaneous adipose tissues and orbicularis oculi muscle (OOM) through SE. The correction of lateral wrinkles, such as crow's feet, can resolve the problem of skin overhanging due to differences in skin thickness, make pre-existing double eyelids look more natural, and solve the difficulty of sagging eyelids in patients who do not desire double eyelids. Moreover, this approach can be used in cases of previous subbrow incision scar or to change the position of an eyebrow tattooing. However, this approach also has problems of eyebrow descend or flattening, and shortening of the distance between eyebrow and lid margin. Severe drooping of the medial skin cannot be easily corrected. In addition, periocular sensory abnormality or scar formation may also occur following this procedure [7,1012]. In an effort to complement the advantages and to make up for the disadvantages of this method, clinicians at this department attempted SE-tadpole shaped excision blepharoplasty (TSEB). We report these cases since the procedure engendered an excellent outcome.

\section{METHODS}

This study included 15 patients for a total of 30 eyelids, who had been admitted to this hospital with a chief complaint of droopy eyelids and underwent SE-TSEB from January 2013 to November 2014. Most of these patients had a complaint of visibility difficulties and aesthetic frustration due to blepharochalasis. Among patients admitted to this hospital with a chief complaint of droopy eyelids, SE-TSEB was performed the following cases: 1) patients who showed moderate to severe blepharochasis with severe lateral wrinkles (crow's feet); 2) patients with a history of pre-existent SE blepharoplasty; 3 ) patients with a tattoo on the eyebrows, who want to make revision; and 4) patients with eyelid skins that are thick or have overly protuberant fat pads of the retroorbicularis oculi fat (ROOF), and have a plan to extirpate them. On the other hand, this procedure was not performed for (1) cases where either eyelid ptosis or brow ptosis was severe, and (2) cases in which patients do not desire either any modification of the existing double eyelid line or subbrow scar. Postoperative evaluations were carried out by utilizing the extent of visible scar, periocular sensory abnormality, the extent of correction of the lateral wrinkles, correction of visibility difficulty, and the degree of natural eyelid wrinkles.

\section{Surgical technique \\ Preoperative design}

First, the incision lines for classical upper blepharoplasty for each patient were designed. The amount of stretched skin throughout the eyelid was verified and the pinch test was done with forceps to find the amount of skin excision in order to design the incision line on the pretarsal lid crease. Accepted crescent-shaped blepharoplas- ty is somewhat convex, which is widest at the center of the lid with or without a lateral extension. After measuring the amount of lateral skin excision needed to correct lateral hooding, this measurement was utilized in deciding the extent of excision required for blepharoplasty by making a SE. The maximal vertical width of the subbrow skin to be removed was determined within the range of 8 to $15 \mathrm{~mm}$. The upper incision line was drawn along the lower border of the eyebrow after deciding the amount of skin excision. At this time, the medial starting point of the incision line was based on the medial limbus. Further medial extension of the incision line was disallowed to prevent unfavorable scar in the eye. The lateral end of the incision line was not extended more than $10 \mathrm{~mm}$ from the lateral end of the eyebrow. After drawing the incision line of the upper part of the subbrow skin to be removed, the lower incision line was drawn in a crescent shape depending on the amount of excision required. Then, the incision line of the pretarsal lid crease form a tadpole shape, after removing the subbrow excess skin. Taking advantage of this new design, the excess lid skin in the medial aspect and the excessive lateral wrinkles could be effectively removed (Fig. 1).

\section{Operative technique}

After a patient was allowed to lie down in a supine position, local anesthesia was carried out on the incision line just below the eyebrow and that of the area just above the tarsal plate with $2 \%$ lidocaine/1:100,000 epinephrine solution. Upon adequate induction of local anesthesia, an incision was made to the depth of subcutaneous fat layer, using a No. 15 blade, along the incision line of the upper skin flap in the subbrow area. A beveled incision was made by tilting the blade at approximately a $30^{\circ}$ angle so that the hair follicles would not be damaged. Likewise, en block excision was per-
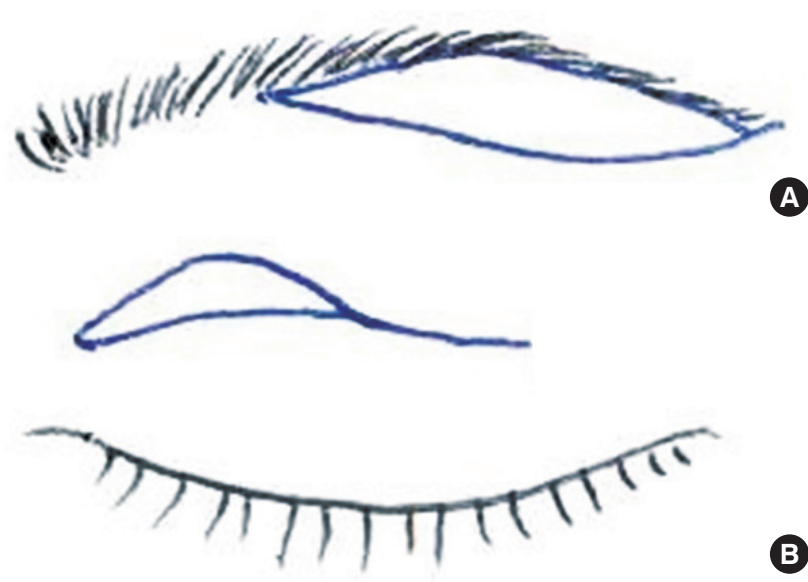

B

Fig. 1. Design of skin excision. (A) Following the lower edge of the eyebrow, from the point A located at the medial limbus, extends to the tail of the eyebrow. (B) Tadpole shaped excision which is widest medially and that tapers to a point B laterally to the point located at the lateral end of eyebrow. 


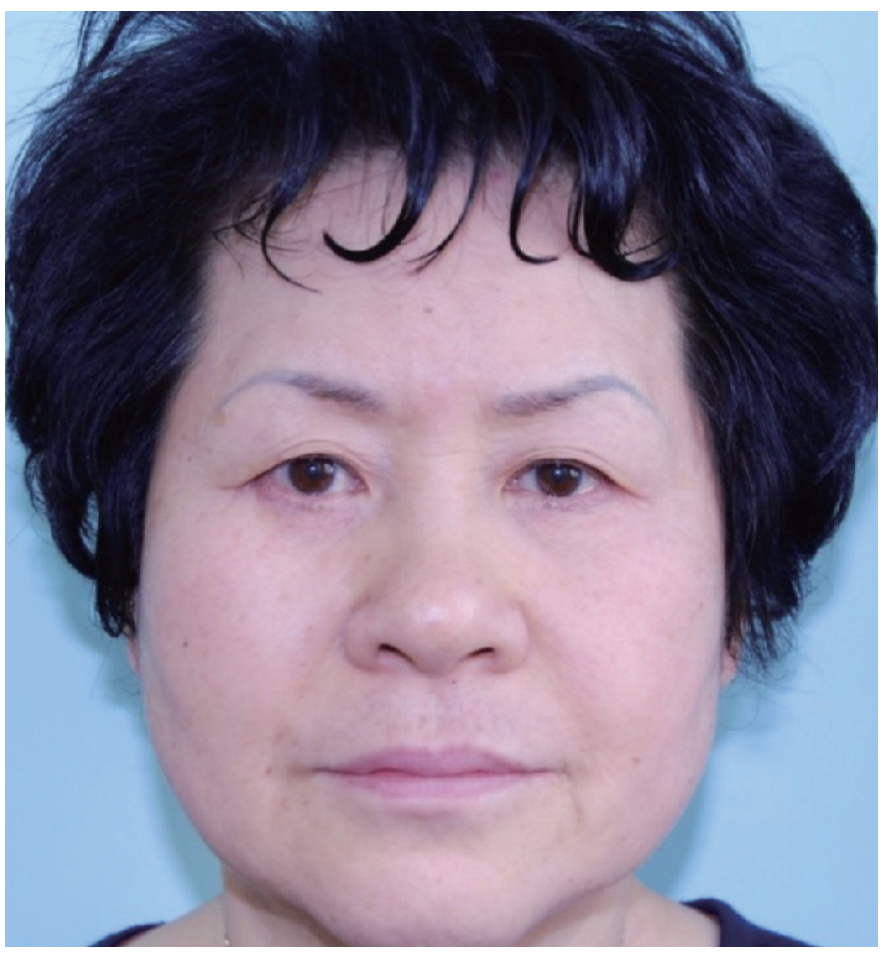

Fig. 2. Preoperative view of a 49 year-old woman.

formed by making an incision on the lower incision line and extirpating the skin and subcutaneous fat on the OOM, excising from the lateral aspect to the medial direction. Hypertrophy of the OOM or protuberant fat underneath the OOM was managed by a partial excision of the muscle or by extirpating the adipose tissues just beneath it. Those incision sites were closed with 6-0 monocryl and 6-0 nylon suture material after all the bleeders were totally controlled by bipolar cauterization. In the same way, the skin and subcutaneous fat layer were extirpated incorporating the design for classical upper blepharoplasty. In certain cases, a part of the OOM was removed and the double eyelids were created; or in cases of blepharoptosis, it was corrected by exposing the levator aponeurosis.

\section{RESULTS}

Among 15 patients, 12 patients were females and 3 were males with a mean age of 55 years. It was their first surgery for 11 patients, while 4 patients had either previous classical upper blepharoplasty or SE blepharoplasty. Patients were observed for any development of hematoma or edema on the postoperative day 2 . The suture material was removed on the 5th day of surgery. The mean duration of follow-up observation was 6 months, while that of surgery was 55 minutes. Two patients did not return to the hospital after removing the suture material. SE-TSEB was effective in patients with droopy eyelids at the severity level of moderate to severe. During the followup observation period, all patients showed amicable correction of

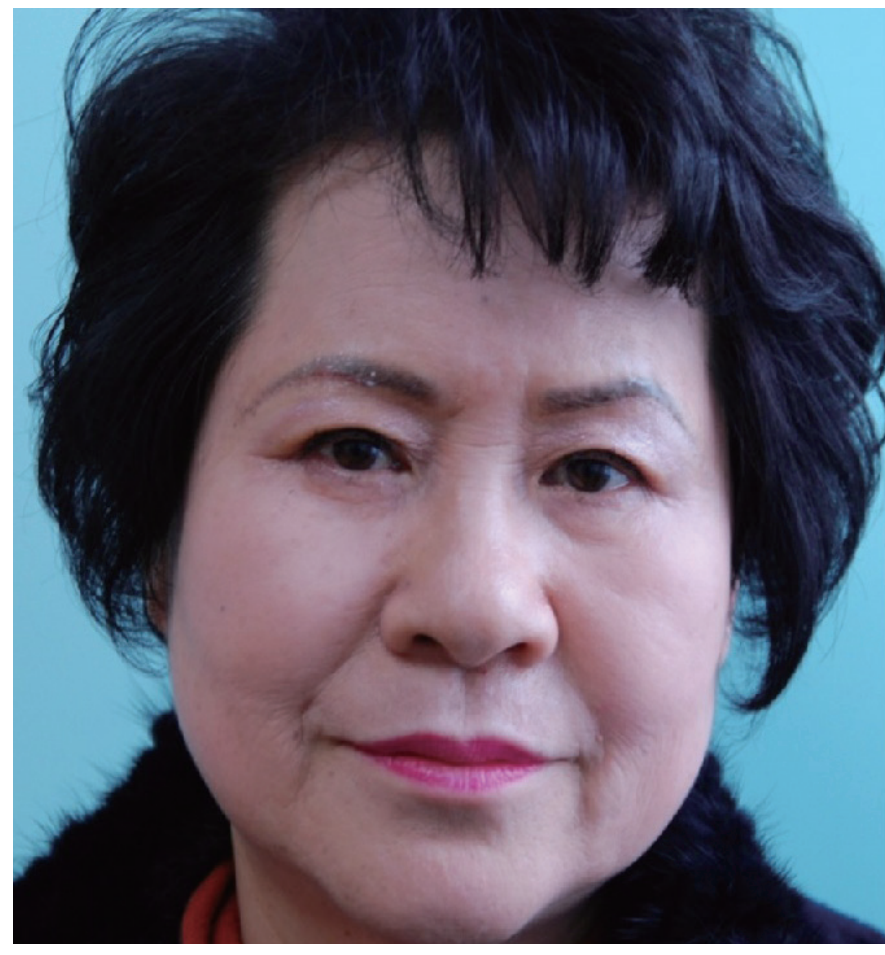

Fig. 3. The 6 months follow-up after Combined subbrow excision with upper blepharoplasty using a tadpole shaped excision for blepharochalasis.

visibility difficulty, of which patients had complaint, and were aesthetically satisfied with the postoperative results with respect to correction of the lateral wrinkles (Fig. 2 and 3). Scars formed in the subbrow incision line were within the scope of management. However, 2 patients had to undergo scar removal procedure by using a silicone gel and a scar laser. Also, 3 patients had complained of periocular sensory abnormality, which, however, improved spontaneously in 5 months after surgery. Problems of edema, wound infection, or wound disruption did not occur either (Table 1).

\section{DISCUSSION}

Characteristic change of the eyelid, as people age, is drooping of the palpebral skin, the subcutaneous fat, or the OOM. Although the skin thickness does not always change in proportion to aging [13], loss of the elastic fibers constituting the skin and skin laxity undergo remarkable alteration as time goes by [14]. Furthermore, histologically, each layer of the OOM does not always undergo the aging process, such as loss of muscular fibers. Nevertheless, solid adhesion between the orbicularis muscle and skin, the subcutaneous fat layer may show structural weakening due to the aging process [15]. Droopy eyelid caused by various changes leads to functional disabilities, such as visibility difficulty for Westerners and Asians, as well as the issue of aesthetic discontent. Thus, the efforts to solve such problem made by plastic surgeons have resulted in 
Table 1. Patients summary

\begin{tabular}{llll}
\hline Sex/Age & Primary/Secondary & \multicolumn{1}{c}{ Surgical treatment } & No \\
\hline F/52 & Primary & SE-TSEB & NO \\
$F / 50$ & Primary & SE-TSEB, ROOF remove, OOM excision & NO \\
$F / 61$ & Secondary & SE-TSEB, Ptosis correction & Sensory abnormality \\
$F / 51$ & Primary & SE-TSEB & Sensory abnormality \\
$F / 49$ & Primary & SE-TSEB, OOM excision & NO \\
$F / 52$ & Primary & SE-TSEB, ROOF remove, OOM excision & Sensory abnormality \\
$F / 62$ & Secondary & SE-TSEB & Scar problem \\
$F / 58$ & Primary & SE-TSEB, Ptosis correction & NO \\
$M / 55$ & Primary & SE-TSEB & Scar problem \\
$F / 66$ & Secondary & SE-TSEB, Ptosis correction & NO \\
$M / 61$ & Primary & SE-TSEB, OOM excision & NO \\
$F / 49$ & Primary & SE-TSEB & NO \\
$F / 62$ & Secondary & SE-TSEB, Ptosis correction & NO \\
$M / 51$ & Primary & SE-TSEB & NO \\
\hline
\end{tabular}

SE-TSEB, subbrow excision-tadpole shaped excision blepharoplasty; ROOF, retroorbicularis oculi fat; 0OM, orbicularis oculi muscle.

technical progression and evolvement throughout various ages. As stated above, the classical upper blepharoplasty, the fundamental approach for the management of a droopy eyelid, involves excisions of the excessive, droopy palpebral skin, subcutaneous fats and OOM through incision lines of the pretarsal and lid crease [1-4]. This surgical procedure has first been devised and became the foundation of upper blepharoplasty for a long time. This has been attributed to the unique advantage of this surgical method. Owing to the aspect of extirpation being done appropriately throughout the entire eyelid for this method, the medial and lateral perspective of the eyelid can display a relatively even appearance. Moreover, accompanied aponeurotic blepharoptosis may also be corrected without an additional procedure $[5,6]$. Patients with or without a pre-existent double eyelid may be provided with new double eyelids or double eyelids with a desired height [15]. This procedure also has limitations and disadvantages, nonetheless, which led to development of other methods that have been devised to improve on these drawbacks. Unnatural appearance after surgery may be burdensome for some patients. Particularly, elderly people may not desire newly-formed coquettish eyes. Besides, as aging progresses, it is known that sagging of the skin may be most severe in the lateral $2 / 3$ of the eyelids [7]. With classical upper blepharoplasty, effective correction of a scar problem may not be easy, and there may be an overhanging problem of protuberant eyelids due to differences in thickness of the pretarsal skin and subbrow skin. Upper blepharoplasty with SE is the procedure devised to improve such limitations of the classical upper blepharoplasty. This procedure, performed through the incision line of the lower border of the eyebrow, may be undertaken without much alteration in facial morphology. A postoperative scar also may not be easily seen by an eyebrow's borderline. The sagging of the lateral wrinkle can be effectively corrected, while a pre-existent eyelid wrinkle can also be managed without making much change. Hence, this is an effective method for elderly patients who do not desire an operational look. Likewise, this approach may be effective in patients with a pre-existent subbrow incision scar, and in patients who want to change the position of an eyebrow tattoo [7-10]. However, this surgical procedure also has its limitations and shortcomings, in that, the drooping of the medial skin cannot be effectively corrected in patients with a problem of eyebrow descend or flattening, shortening of the distance between the eyebrow and lid margin, or severe skin sagging. Since introduction of this procedure by Parkes et al.[9] for the first time, numerous enhanced surgical techniques have been introduced. Widgerow [16] has advocated an extended lateral segmental orbicularis excision concomitant with upper blepharoplasty. Har-Shai and Hirshowitz's [17] extended upper blepharoplasty for lateral hooding of the upper eyelid, using a scalpel-shaped excision, places more emphasis on the importance of debulking the thick skin and subcutaneous fat laterally, to obtain a pleasing postoperative open appearance to the eyes. Likewise, each approach to upper blepharoplasty has its merits and drawbacks. An excellent result may be achieved by selecting a surgical technique that is suitable for the characteristics of a patient. Clinicians at this department considered a treatment modality that utilizes two surgical procedures performed simultaneously in an attempt to overcome the limitations of each method. SE blepharoplasty has a limitation in that, this procedure cannot be used in patients with severe skin drooping of the eyelid. Particularly in patients with a medial wrinkle drooping of the eyelid. On the other 
hand, classical upper blepharoplasty has a limitation in making corrections of the excessive lateral wrinkles. On the idea of combination of these two surgical approaches, more effective upper blepharoplasty may be undertaken in patients with relatively severe blepharochalasis. Owing to the fact that each procedure is not so complicated and time consuming, simultaneous performance of these two methods could easily be completed within one hour or so, and does not require technical expertise. Also, the amount of SE was determined by measuring the excessive lateral skin to be excised after the classical upper blepharoplasty had been designed before surgery. Thus, the problem of lagophthalmos by excessive extirpation did not occur, and the droopy skin of the lateral aspect of the eyelids was effectively corrected by using the SE. However, dog-ear, generated by the difference in the excised amount of the medial and lateral aspects, and the tension due to the upper and lower suturing of the eyelid following excessive extirpation, leads to an unnatural appearance of the skin. However, simultaneous excisions of two areas of the OOM may provide more natural looking eyes by natural relocation of the musculo-cutaneous composite tissues between the excised areas of the upper and lower excised area. Besides, owing to the aspect that the excision was simultaneously carried out in two areas, the protuberant fat pad beneath the OOM could easily be removed through the subbrow incision line, while it has an advantage that ptosis correction could concurrently be performed in patients accompanied by aponeurotic blepharoptosis.

\section{CONCLUSIONS}

The consideration is that the surgical approach implemented by clinicians at this department is a treatment modality that overcomes the disadvantages and limitations of classical upper blepharoplasty and SE blepharoplasty. Furthermore, maximizing the advantage of each surgical approach is another innovative approach for correction of changes in the upper eyelid, induced by aging.

\section{REFERENCES}

1. Rohrich RJ, Coberly DM, Fagien S, et al. Current concepts in aesthetic upper blepharoplasty. Plast Reconstr Surg 2004;113:32e-42e.
2. McCurdy JA, Jr. Upper blepharoplasty in the Asian patient: the "double eyelid” operation. Facial Plast Surg Clin North Am 2005;13:47-64.

3. Karam AM, Lam SM. Management of the aging upper eyelid in the asian patient. Facial Plast Surg 2010;26:201-8.

4. Kim DW, Bhatki AM. Upper blepharoplasty in the Asian eyelid. Facial Plast Surg Clin North Am 2007;15:327-35, vi.

5. de la Torre JI, Martin SA, De Cordier BC, et al. Aesthetic eyelid ptosis correction: a review of technique and cases. Plast Reconstr Surg 2003; 112:655-60; discussion 61-2.

6. Carraway JH, Tran P. Blepharoplasty with ptosis repair. Aesthet Surg J 2009;29:54-61.

7. Kim YS, Roh TS, Yoo WM, et al. Infrabrow excision blepharoplasty: applications and outcomes in upper blepharoplasty in Asian women. Plast Reconstr Surg 2008;122:1199-205.

8. Murakami M, Hyakusoku H. Treatment of upper eyelid dermatochalasis by resection of excess skin at the inferior margin of the eyebrow. J Nippon Med Sch 2008;75:364-6.

9. Parkes ML, Kamer FM, Merrin ML. Infrabrow lift. Laryngoscope 1976; 86:1869-72.

10. Lee D, Law V. Subbrow blepharoplasty for upper eyelid rejuvenation in Asians. Aesthet Surg J 2009;29:284-8.

11. Sugamata A, Yoshizawa N. Infraeyebrow excision blepharoplasty for Japanese blepharochalasis: review of 35 patients over 60 years old. Scand J Plast Reconstr Surg Hand Surg 2010;44:17-20.

12. Hara T, Hara T, Narita M, et al. Infero-eyebrow blepharoplasty for the upper eyelids of elderly patients. Br J Ophthalmol 2011;95:109-11.

13. Hwang K, Kim DJ, Kim SK. Does the upper eyelid skin become thinner with age? J Craniofac Surg 2006;17:474-6.

14. Pottier F, El-Shazly NZ, El-Shazly AE. Aging of orbicularis oculi: anatomophysiologic consideration in upper blepharoplasty. Arch Facial Plast Surg 2008;10:346-9.

15. Hoorntje LE, Lei B, Stollenwerck GA, et al. Resecting orbicularis oculi muscle in upper eyelid blepharoplasty--a review of the literature. J Plast Reconstr Aesthet Surg 2010;63:787-92.

16. Widgerow AD. Upper blepharoplasty with lateral segmental orbicularis excision. Ann Plast Surg 2003;50:471-4.

17. Har-Shai Y, Hirshowitz B. Extended upper blepharoplasty for lateral hooding of the upper eyelid using a scalpel-shaped excision: a 13-year experience. Plast Reconstr Surg 2004;113:1028-35; discussion 36. 